\title{
Magnetic dipolar ordering and hysteresis of geometrically defined nanoparticle clusters
}

\author{
Kure, Mathias; Beleggia, Marco; Frandsen, Cathrine
}

Published in:

Journal of Applied Physics

Link to article, DOI:

$10.1063 / 1.4994002$

Publication date:

2017

Document Version

Publisher's PDF, also known as Version of record

Link back to DTU Orbit

Citation (APA):

Kure, M., Beleggia, M., \& Frandsen, C. (2017). Magnetic dipolar ordering and hysteresis of geometrically defined nanoparticle clusters. Journal of Applied Physics, 122(13), [133902 ]. https://doi.org/10.1063/1.4994002

\section{General rights}

Copyright and moral rights for the publications made accessible in the public portal are retained by the authors and/or other copyright owners and it is a condition of accessing publications that users recognise and abide by the legal requirements associated with these rights.

- Users may download and print one copy of any publication from the public portal for the purpose of private study or research.

- You may not further distribute the material or use it for any profit-making activity or commercial gain

- You may freely distribute the URL identifying the publication in the public portal 


\section{Magnetic dipolar ordering and hysteresis of geometrically defined nanoparticle clusters}

Mathias Kure, Marco Beleggia, and Cathrine Frandsen

Citation: Journal of Applied Physics 122, 133902 (2017); doi: 10.1063/1.4994002

View online: http://dx.doi.org/10.1063/1.4994002

View Table of Contents: http://aip.scitation.org/toc/jap/122/13

Published by the American Institute of Physics

\section{Articles you may be interested in}

Influence of magnetization on the applied magnetic field in various AMR regenerators

Journal of Applied Physics 122, 133901 (2017); 10.1063/1.4986936

Investigation of magnetic and magneto-transport properties of ferromagnetic-charge ordered core-shell nanostructures

Journal of Applied Physics 122, 134301 (2017); 10.1063/1.4993095

$\mathrm{CaFe}_{2} \mathrm{O}_{4}$ as a self-sufficient solar energy converter

Journal of Applied Physics 122, 133114 (2017); 10.1063/1.4993537

Effect of Holmium substitution on the magnetic and magnetodielectric properties of multiferroic $\mathrm{Bi}_{2} \mathrm{Fe}_{4} \mathrm{O}_{9}$ Journal of Applied Physics 122, 134103 (2017); 10.1063/1.4994645

Investigation of low temperature quantum crossover in Josephson junctions

Journal of Applied Physics 122, 133904 (2017); 10.1063/1.4986757

Magnetic properties and evidence of current-induced perpendicular field in epitaxial ferrimagnetic $\mathrm{Mn}_{4} \mathrm{~N}(002)$

film mixed with (111) phase

Journal of Applied Physics 122, 133905 (2017); 10.1063/1.5006449

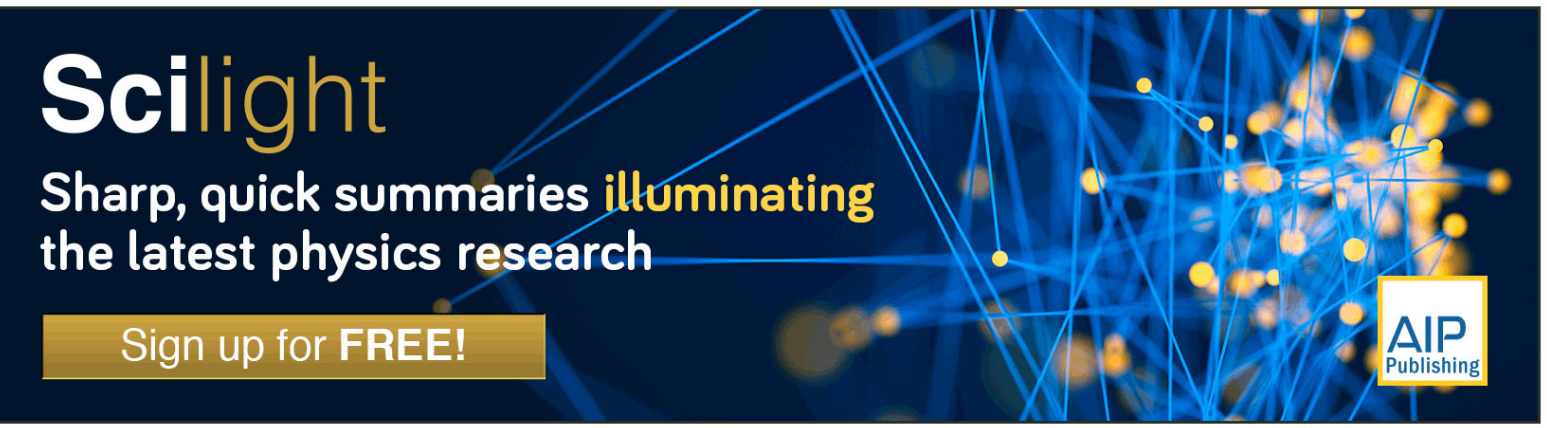




\title{
Magnetic dipolar ordering and hysteresis of geometrically defined nanoparticle clusters
}

\author{
Mathias Kure, ${ }^{1, a)}$ Marco Beleggia $^{2}$ and Cathrine Frandsen ${ }^{1}$ \\ ${ }^{1}$ Department of Physics, Technical University of Denmark, DK-2800 Kgs. Lyngby, Denmark \\ ${ }^{2}$ Center for Electron Nanoscopy, Technical University of Denmark, DK-2800 Kgs. Lyngby, Denmark
}

(Received 3 July 2017; accepted 19 July 2017; published online 3 October 2017)

\begin{abstract}
Magnetic nanoparticle clusters have several biomedical and engineering applications, and revealing the basic interplay between particle configuration and magnetic properties is important for tuning the clusters for specific uses. Here, we consider the nanoparticles as macrospins and use computer simulations to determine their magnetic configuration when placed at the vertices of various polyhedra. We find that magnetic dipoles of equal magnitude arrange in flux-closed vortices on a layer basis, giving the structures a null remanent magnetic moment. Assigning a toroidal moment to each layer, we find that the geometrical arrangement, i.e., "triangular packing" vs. "square packing," of the moments in the adjacent layer determines whether the flux-closed layers are ferrotoroidal (co-rotating vortices) or antiferrotoroidal (counter-rotating vortices). Interestingly, upon adding a single magnetic moment at the center of the polyhedra, the central moment relaxes along one of the principal axes and induces partial alignment of the surrounding moments. The resulting net moment is up to nearly four times that of the single moment added. Furthermore, we model quasi-static hysteresis loops for structures with and without a central moment. We find that a central moment ensures an opening of the hysteresis loop, and the resultant loop areas are typically many-fold larger compared to the same structure without a central moment. Published by AIP Publishing. [http://dx.doi.org/10.1063/1.4994002]
\end{abstract}

\section{INTRODUCTION}

A proposed avenue to construct nanomaterials featuring some desired magnetic property is to assemble them bottomup, utilizing nanoparticles as building blocks. The resulting assemblies represent a wide range of structures, such as clusters, ${ }^{1}$ rings, ${ }^{2}$ chains, ${ }^{3}$ dendrites, ${ }^{4}$ and closely packed $3 \mathrm{D}$ structures. ${ }^{5}$ These materials are currently being investigated for applications as diverse as permanent magnets, ${ }^{6}$ data storage, ${ }^{7}$ magnetic sensors, ${ }^{8}$ and for therapeutic uses such as drug carriers and agents of hyperthermia. ${ }^{9}$

Their overall magnetic properties, e.g., net moment and coercivity, depend non-trivially on the internal configurational space of magnetic nanoparticles, i.e., mutual arrangement, morphology, composition, and particle size. Clearly, the resulting magnetic properties are decisive for their applications. For instance, in the case of magnetic hyperthermia, the heating properties of magnetic nanomaterials are positively linked to the hysteresis, ${ }^{10}$ while for use in sensors or power converters hysteresis losses have to be limited.

So far, most modeling studies of nanoparticle-materials have focused on understanding the structure-property link of large 3D structures, e.g., Refs. 11-13, and 1-2D structures, e.g., Refs. 14 and 15, while small 3D structures such as clusters have been investigated only sparingly, e.g., Refs. 16 and 17. However, due to a large number of biomedical applications of magnetic nanoparticle clusters, see e.g., Refs. 1 and 9, computer simulations, which seek to replicate the experimentally observed properties begin to be reported e.g., Refs. 16 and 18 . Yet, the causal link between the configurational space and the

${ }^{a)}$ Electronic mail: matku@fysik.dtu.dk desired emerging property in nanoparticle clusters is not yet fully established, ${ }^{19}$ and a more fundamental overview seems to be needed. In the case of small 3D-structures with often a complex internal particle arrangement, the structure-property link can be difficult to "grasp," as relatively small variations in configurational space and interparticle interactions may lead to very different properties. It therefore appears relevant to address the fundamentals and question which magnetic order does even very simple nanoparticle clusters, e.g., particles arranged as a tetrahedron or cube, possess, and to which extent would it be possible to tune the properties by structural design.

In this paper, we examine small geometrically defined nanoparticle clusters, consisting of 4-20 magnetic moments placed at the vertices of various polyhedra. The nanoparticles are modeled as Heisenberg macrospins. The clusters considered are the five Platonic solids (tetrahedron, octahedron, cube, dodecahedron, icosahedron) and the square antiprism. We also examine the same structures with a central moment added [transforming the cube into a body centered cubic (bcc) structure, etc.]. We determine the dipolar ground states of these clusters, and systematically discuss these states, as well as the role of the added central moment in enhancing the total moment and hysteresis heating.

\section{METHOD}

We model collections of macrospins as pure magnetic dipole moments locked in spherical particles (corresponding to infinite magnetocrystalline anisotropy), and assume that their interaction is purely dipolar. The interaction energy of this system is described by 


$$
U=\frac{\mu_{0}}{4 \pi} \sum_{i<j}\left(\frac{\vec{\mu}_{i} \cdot \vec{\mu}_{j}}{r_{i j}^{3}}-3 \frac{\left(\vec{\mu}_{i} \cdot \vec{r}_{i j}\right)\left(\vec{\mu}_{j} \cdot \vec{r}_{i j}\right)}{r_{i j}^{5}}\right),
$$

where $\vec{\mu}_{i}$ is the magnetic moment of macrospin $i$ and $\vec{r}_{i j}$ is the separation vector between macrospins $i$ and $j$. We assign to each macrospin, a magnetic moment of identical magnitude $\left|\vec{\mu}_{i}\right|=\mu$. This leads to the effective interaction

$$
\begin{gathered}
U=U_{0} \sum_{i<j}\left(\frac{\hat{\mu}_{i} \cdot \hat{\mu}_{j}-3\left(\hat{\mu}_{i} \cdot \hat{r}_{i j}\right)\left(\hat{\mu}_{j} \cdot \hat{r}_{i j}\right)}{\left|r_{s, i j}^{3}\right|}\right), \\
U_{0}=\frac{\mu_{0} \mu^{2}}{4 \pi a^{3}},
\end{gathered}
$$

where $r_{s, i j}=r_{i j} / a$ and $a$ is the center-to-center distance between two nearest-neighbor moments.

To determine the ground state energies and configurations, we perform Molecular Dynamics (MD) simulations on the collections of moments, with moment positions stationary, such that rotation of the moments in three dimensions are the only available degrees of freedom. MD has been chosen over energy-minimizing algorithms to allow for displacement of the moments at a later stage.

The rotation of spherical particles is modeled by Newton's equations of motion for a spinning body. We calculate the torque $\vec{\tau}_{i}$ on a macrospin $\vec{\mu}_{i}$ from its interaction with the field $\vec{B}_{i}$, i.e., the superposition of the dipole fields from the other macrospins, as

$$
\begin{gathered}
\vec{\tau}_{i}=\vec{\mu}_{i} \times \vec{B}_{i}\left(r_{i}\right), \\
\vec{B}_{i}\left(r_{i}\right)=\frac{B_{0}}{2} \sum_{j \neq i}\left(\frac{3\left(\hat{\mu}_{j} \cdot \hat{r}_{i j}\right) \hat{r}_{i j}-\hat{\mu}_{j}}{\left|r_{s, i j}^{3}\right|}\right),
\end{gathered}
$$

where $B_{0}$ is $U_{0} / \mu$. For simulations of hysteresis properties, we apply an external magnetic field with field strength $B_{\text {ext }}$ generating an additional torque on the $i$ th moment, in which case $\vec{B}_{i}\left(r_{i}\right)$ is replaced by $\vec{B}_{i}\left(r_{i}\right)+\vec{B}_{\text {ext }}$ in Eq. (4).

For the integration of the equations of motion, we use the velocity Verlet algorithm described by ${ }^{20}$

$$
\begin{aligned}
\vec{\theta}(t+\delta t) & =\vec{\theta}(t)+\delta t \vec{\omega}(t)+\frac{1}{2}(\delta t)^{2} \vec{\alpha}(t), \\
\vec{\omega}(t+\delta t) & =\vec{\omega}(t)+\frac{1}{2} \delta t[\vec{\alpha}(t)+\vec{\alpha}(t+\delta t)],
\end{aligned}
$$

where $\vec{\theta}, \vec{\omega}$, and $\vec{\alpha}$ are the angular displacement, velocity and acceleration, respectively. A midpoint angular velocity is calculated at time $t+\frac{1}{2} \delta t$, and this is used to advance the position to time $t+\delta t$, from where a new calculation of the torque and angular acceleration is performed. This angular acceleration in turn is used to advance the angular velocity to the current time-step. This algorithm is computationally simple and is furthermore symplectic such that there is no artificial damping or excitation. ${ }^{21}$

The simulations are initialized with moments at rest and random starting orientations in all three dimensions.

The spatial rotations resulting from the angular accelerations are performed by quaternion algebra to avoid the usual problems associated with rotation in three dimensions. ${ }^{22}$ The simulation terminates when the largest angular displacement between successive iterations falls below a pre-determined limit. The simulations are repeated several hundred times from different starting configurations to substantiate that the macrospins relax towards the ground state rather than towards a local minimum of the energy landscape. To further quantify this, the energies of selected moment configurations have been compared. Since we are working with a relatively small $(\leq 20)$ number of moments in simple geometrical structures, we expect to find only a few well-defined states as ground state candidates.

For hysteresis simulations, we start from a ground state of the selected structure and stepwise apply the $B_{\text {ext }}$ field along a fixed direction. At each step, the moments of the structure are allowed to relax. The simulation terminates after completing a full hysteresis loop.

\section{RESULTS AND DISCUSSION}

\section{A. 2D polygons}

In order to introduce our nomenclature, we first look at dipoles located at the vertices of regular convex 2D polygons. In such cases, we find as observed by several others that the dipolar interactions will relax the moments into fluxclosed vortex structures. The equilibrated magnetic moments are directed tangentially to the circumscribed circle of the polygon, and the resulting net moment is zero. ${ }^{23}$ Such a fluxclosed vortex structure for an equilateral triangle, with qualitative field contours superposed for illustrative purposes, is shown in Fig. 1. The rotation of the vortices is either clockwise $(\mathrm{CW})$ or counterclockwise $(\mathrm{CCW})$, hence the energy ground state is twice degenerate.

For each polygon, a toroidal moment can be defined as

$$
\vec{T}=\frac{1}{2} \sum_{i=1}^{n} \vec{r}_{i} \times \vec{\mu}_{i},
$$

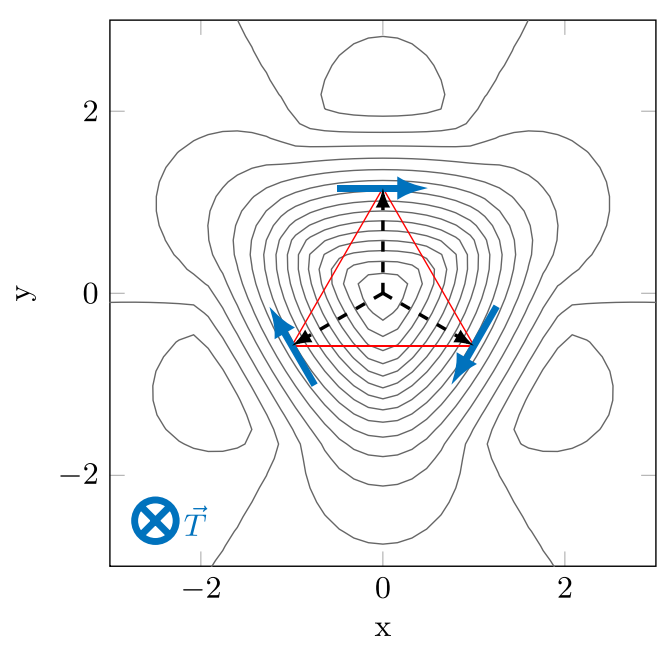

FIG. 1. The ground state of three magnetic moments (blue arrows) placed at the vertices of an equilateral triangle (red) and the $\vec{r}_{i}$ vectors (black dashed arrows) from the polygon center to the vertices. The moments organize to form a flux-closed vortex, shown here as rotating clockwise and giving rise to an into-page toroidal moment $\vec{T}$. The qualitative field contours produced by the three moments are also shown. 
where $\vec{r}_{i}$ is the position vector from the origin to the $i$-th moment. The toroidal moment is origin independent, ${ }^{24}$ only if $\vec{\mu}_{\text {tot }}=0$. Figure 1 shows the $\vec{r}_{i}$ and $\vec{\mu}_{i}$ vectors. The toroidal moment (which points into the page in Fig. 1) represents the sense of rotation of the flux-closed vortex. For an $n$-sided regular convex polygon, we define a maximal toroidal moment $T_{0}=n \mu a / 2 \sin (\pi / n)$, associated with the perfect vortex state where all the moments are tangential; $T_{0}$ is to be understood as a saturation toroidal moment.

\section{B. Platonic structures}

The 3D structures - the tetrahedron, octahedron, cube, square antiprism, dodecahedron, and icosahedron-shown in Fig. 2 can all be described as composed of layers of convex regular polygons with their centers aligned on top of each other. The shape of the polygon layers are typically the same as the shape of the polygon faces, i.e., the octahedron and the icosahedron both have triangular layers, whilst the cube has square layers, and the dodecahedron has pentagonal layers. However, in the case of the tetrahedron, which has triangular faces, the layers are described by a segment (a degenerate 2 -sided polygon). The tetrahedron, the octahedron, the cube, and the antiprism each consist of two polygon layers, while the dodecahedron and the icosahedron consist of four layers. We classify the layers as inner and outer layers dependent on whether they have two or one neighboring layers, respectively. For the icosahedron and the dodecahedron, there is a difference in the side-length of the inner and outer polygon layers.

For each of the polyhedra, we identified the ground states for magnetic moments placed at their vertices. The moment configurations of the ground states are shown in Fig. 2, and their magnetic characteristics are listed in Table I. We find
TABLE I. Ground state characteristics of different structures that have been simulated. The type of polyhedra, number of magnetic moments $n$, ground state energy $U$, net moment $\mu_{\text {net }}$, net moment when a central moment of $\mu$ is added $\mu_{\text {net }}^{c}$, maximum toroidal moment $\vec{T}_{0}$, toroidal moment along the $\hat{z}$ direction $\vec{T}_{z}$, and toroidal moment when a central moment of $\mu$ is added $\vec{T}_{z}^{c}$. The values refer to the ground states shown in Fig. 2.

\begin{tabular}{lccccccc}
\hline \hline Type & $n$ & $U / U_{0}$ & $\mu_{\text {net }}(\mu)$ & $\mu_{\text {net }}^{c}(\mu)$ & $\vec{T}_{0}(\mu \cdot a)$ & $\vec{T}_{z}(\mu \cdot a)$ & $\vec{T}_{z}^{c}(\mu \cdot a)$ \\
\hline Tetrahedron & 4 & -0.6250 & 0 & 2.22 & 2 & 2 & 0 \\
Octahedron & 6 & -1.2576 & 0 & 3.70 & $2 \cdot \sqrt{3}$ & $2 \cdot \sqrt{3}$ & 0 \\
Cube & 8 & -1.8154 & 0 & 1.92 & $4 \cdot \sqrt{2}$ & 0 & 0 \\
Square & 8 & -1.9910 & 0 & 2.54 & $4 \cdot \sqrt{2}$ & $4 \cdot \sqrt{2}$ & $4.9871^{\mathrm{a}}$ \\
antiprism & & & & & & & \\
Icosahedron & 12 & -3.1662 & 0 & 1.60 & 9.0691 & 9.0691 & 8.8531 \\
Dodecahedron & 20 & -5.0047 & 0 & 0.69 & 22.2703 & 5.2573 & 5.2514 \\
\hline \hline
\end{tabular}

${ }^{\mathrm{a}}$ The antiprism does not relax to a ground state with the toroidal moment in the $\hat{z}$ direction.

that for all structures, there is a tendency for the ground state to have moments organized into flux-closed vortices on a per-layer basis, where moment components are exclusively within the plane of the layer. Consequently, we find that all the ground states in Fig. 2 have zero net moment.

The rotation $(\mathrm{CW} / \mathrm{CCW})$ of the flux-closed vortex for each layer is shown in the panels of Fig. 2 with a teal arrow. The rotation can change from layer to layer, as it does for the cube and for the dodecahedron, or be the same, as for the tetrahedron, the octahedron, the square antiprism, and the icosahedron. All structures in Fig. 2 have been oriented such that their flux-closed vortices are in the $x-y$ plane. The toroidal moment vector is thus perpendicular to this plane, and a $\mathrm{CW}(\mathrm{CCW})$ rotation gives a negative $\hat{z}$ (positive $\hat{z}$ ) toroidal moment. Ground states that correspond to flux-closing vortex

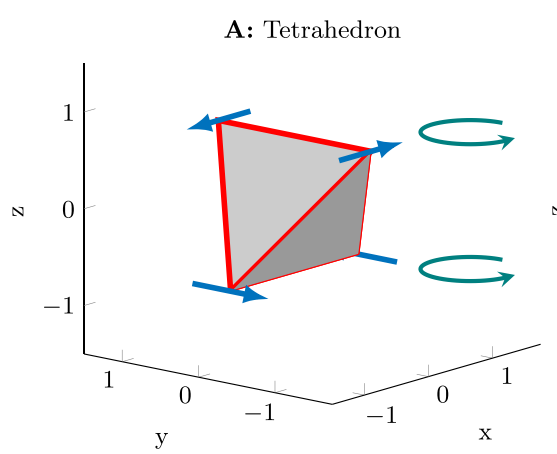

D: Square antiprism
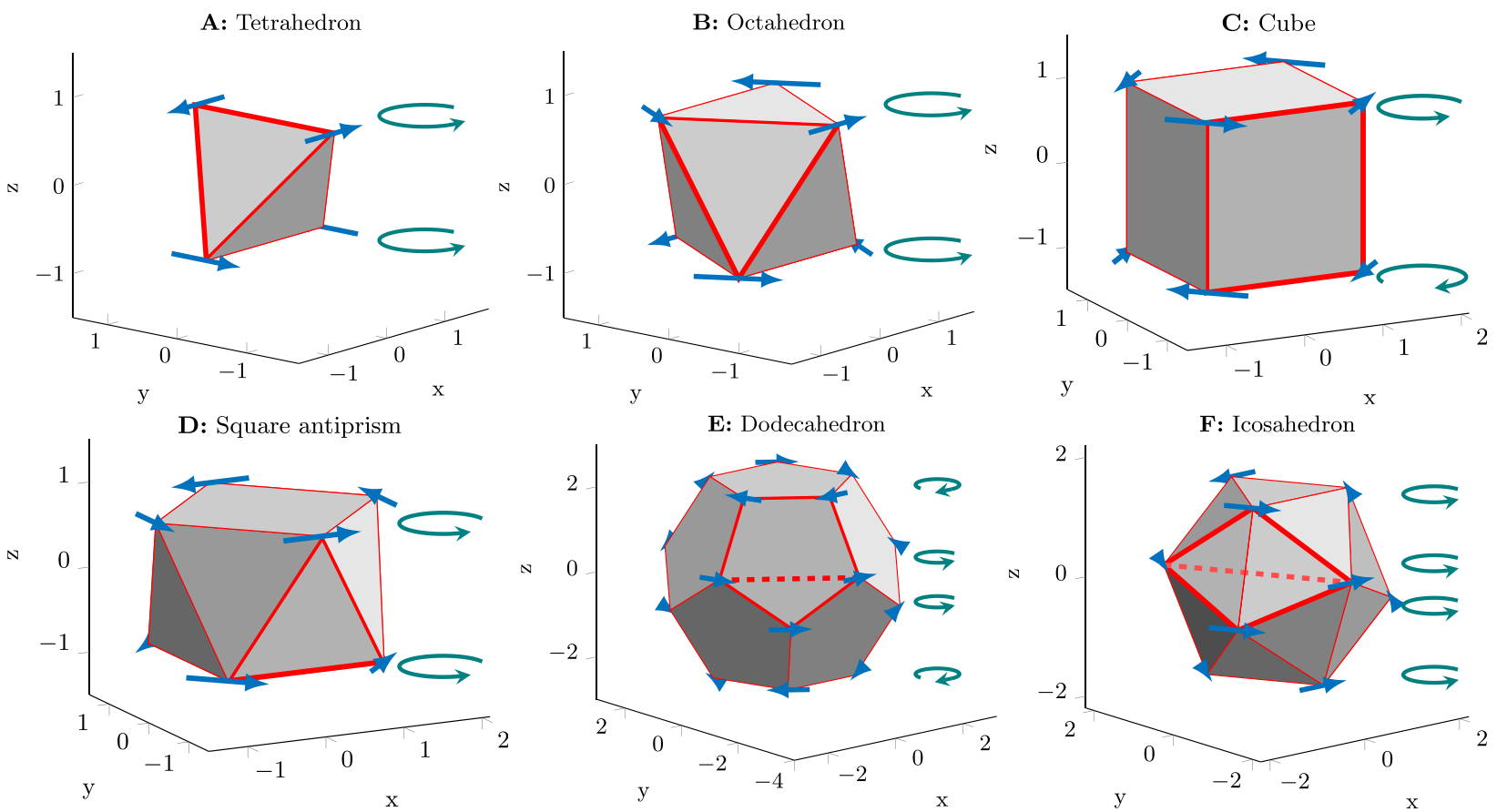

FIG. 2. The dipolar ground states for the: (a) tetrahedron, (b) octahedron, (c) cube, (d) square antiprism, (e) dodecahedron, and (f) icosahedron are indicated with blue arrows showing the relaxed moments at each vertex. For each layer, the rotation is indicated by a teal arrow, such that a $\mathrm{CW}$ (CCW) arrow (as seen along $-\hat{z}$ ) indicates a toroidal moment in the negative $\hat{z}$ (positive $\hat{z}$ ) direction. The packing of the moments is shown with a bold red line, while dashed red lines signify that the packing is not coinciding with the edges of the structure. 
layers in other symmetrically equivalent planes are also possible.

In the case of the tetrahedron, where the moments are only spanned by a line, the resulting moment configuration can still be regarded as flux-closed, since the two moments are oriented tangentially to the circumscribed circle and have the maximum value of the toroidal moment, $\vec{T}=a \mu \hat{z}$. This is, however, not the minimum-energy configuration of two moments on a line.

By comparing $\vec{T}_{z}$ with $\vec{T}_{0}$ (Table I), we see that for the structures that consist of only two layers, the toroidal moment is either at its maximum value or zero. For the dodecahedron with four layers, we find an intermediate value. Two-layer structures have equal magnitude toroidal moment vectors for the two layers, such that co-rotating moments double and counterrotating moments cancel the overall toroidal moment. For multilayer structures, the difference in polygon side-lengths means that the maximum toroidal moments of the single layers have different values. For the dodecahedron, the two larger pentagons co-rotate, giving a net toroidal moment pointing in the direction dictated by the rotation of large pentagons.

Our simulations additionally confirm an interesting finding $^{25}$ for the cube, where the dipolar ground state is found to be infinitely degenerate, with all moments of the degenerate ground states in the planes perpendicular to the cube body diagonals, i.e., the $\{111\}$ planes. These planes are indicated by green circles in Fig. 3, along with 50 sets of moments that have relaxed to the ground states from a random initial orientation. All moments placed body-diagonally opposite to each other are parallel. The moments within a layer have alternating orientations, pointing up and down relative to the z-axis one after the other by the same angle within the $\{111\}$ planes, in all cases leading to zero net moment. We do not find this infinite planar degeneracy in the other structures.

The ground state of the cube presented in Fig. 2(c) has a maximum toroidal moment for a single layer, while the toroidal moment for a layer in Fig. 3 decreases as the moments are rotated out of the $x-y$ plane. The body-diagonally parallel moments ensures that the two layers always have the same magnitude toroidal moment. Since the degenerate ground states retain their counter-rotating nature, the toroidal moment vectors thus cancel out each other.

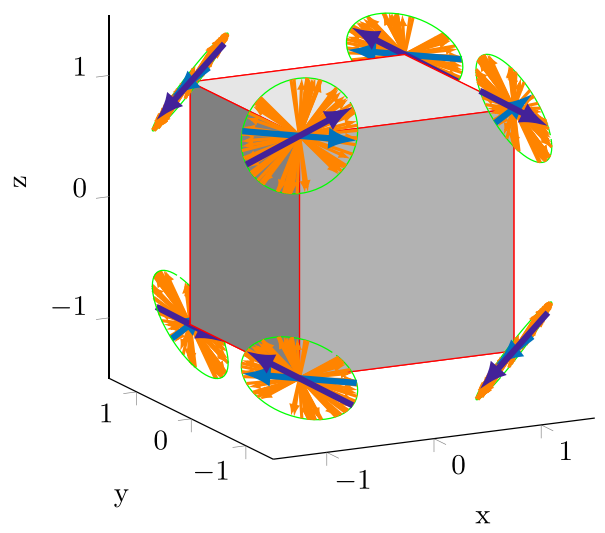

FIG. 3. A collection of 50 of the infinitely degenerate ground states (orange arrows) for the cube, obtained from random starting moment directions. Light blue arrows indicate the ground state presented in Fig. 2(c), while dark blue arrows are guide to the eye for another single set of degenerate moments to better illustrate the alignment of the individual moments.

\section{Effect of packing on the toroidal moment}

We noticed the changing toroidal moment directions for adjacent layers and find that this ferro-/antiferro-toroidicity relates to the packing of the moments in adjacent layers. This becomes very clear when comparing the cube and the square antiprism, both structures with eight moments and the same inter-moment distance, the only difference being that the upper face of the square antiprism has been rotated $45^{\circ}$ with respect to the lower face. As can be seen from the faces highlighted in bold lines in Figs. 2(c) and 2(d), these two structures have two distinct packings between layers: "triangular" or "square."

For all simulated structures, we find that a triangular packing of moments in adjacent layers leads to the co-rotational alignment of moments between layers, while for square packing, the layers are counter-rotating. A combined example is that of the dodecahedron [Fig. 2(e)], which has both squarelike and triangular packings between layers, and consequently changes rotation such that the outer layers are counter-rotating with respect to the inner layers and the two inner layers are co-rotating with respect to each other. This is reminiscent of (anti)ferrotoroidicity, ${ }^{26,27}$ where toroidal moments are (anti)aligned over long distances. For planar structures, it has been previously shown that dipolar forces lead to ferro- or antiferromagnetic alignment dependent on the packing of the moments involved, ${ }^{28}$ and our simulations show that this is also the case of 3D structures, as shown in Fig. 2.

In contrast to ordinary exchange-mediated ferromagnetism where the alignment is global, at least within a rather large domain, it is noticeable that the exchange-decoupled macrospins within the simulated structures can reverse direction from layer to layer.

\section{2D and 3D structures with central moments}

If a central moment is added at the center of a planar structure as shown for the equilateral triangle in Fig. 4, we find that the central moment chooses to align with one of the symmetry axes in the triangle and forces the vertex moment on this axis

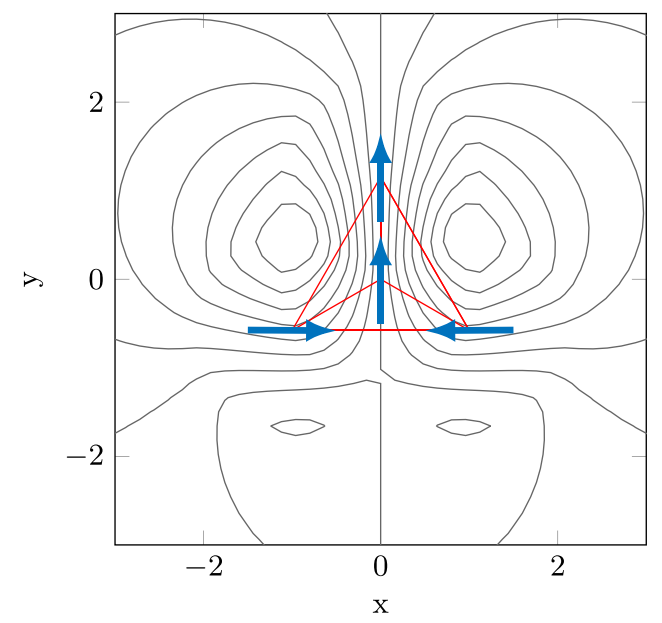

FIG. 4. The ground state of four magnetic moments (blue arrows) placed at the vertices of an equilateral triangle (red) and at the center of mass. The qualitative magnetic contours produced by the four moments are also shown. 
to co-align. The remaining two vertex moments are slightly canted with respect to the base of the triangle, such that the net moment for the structure is $1.99 \mu$ in the direction of the central moment. For polygons with $n>3$ sides (i.e., square, pentagon, hexagon, etc.), we similarly obtain preferred directions of the central moment. When $n$ is even, the central moment has $n$ degenerate orientations (towards the sides for the square and towards the vertices for the hexagon), while when $n$ is odd, it has $2 n$ degenerate orientations. However, coupling between the central moment and the moments on the polygon drops rapidly with increasing $n$, in particular, as $n^{-6}$, and already for $n>8$, the central moment is effectively decoupled and does not relax towards any particular direction over the time scale spanned by our simulations. The rapid decrease of the coupling as $n^{-6}$ originates from the distance between central moment and vertex moments, equal to $a / \sin (\pi / n)$, i.e., the circumradius of the polygon, and from the induced dipole moment on the polygon being proportional to the dipole field of the central moment, each reducing the dipole-dipole energy scale by a factor $n^{-3}$.

We now again turn our attention to the $3 \mathrm{D}$ structures that we previously found to be constructed from layers of polygons. First, we add a central moment to the cube, i.e., changing it into a $b c c$ structure as shown in Fig. 5(a). In this case, the central moment direction is chosen from one of the $\langle 100\rangle$ directions and the infinite degeneracy described above is lifted, leaving a 6-fold degeneracy. The vertex moments are displaced with respect to the layered cube ground state as presented in Fig. 2(c), and acquires a component in the direction of the central moment. In Fig. 5(b), this is a $\mu_{z}$ component of $0.115 \mu$, giving the entire structure a net magnetic moment of
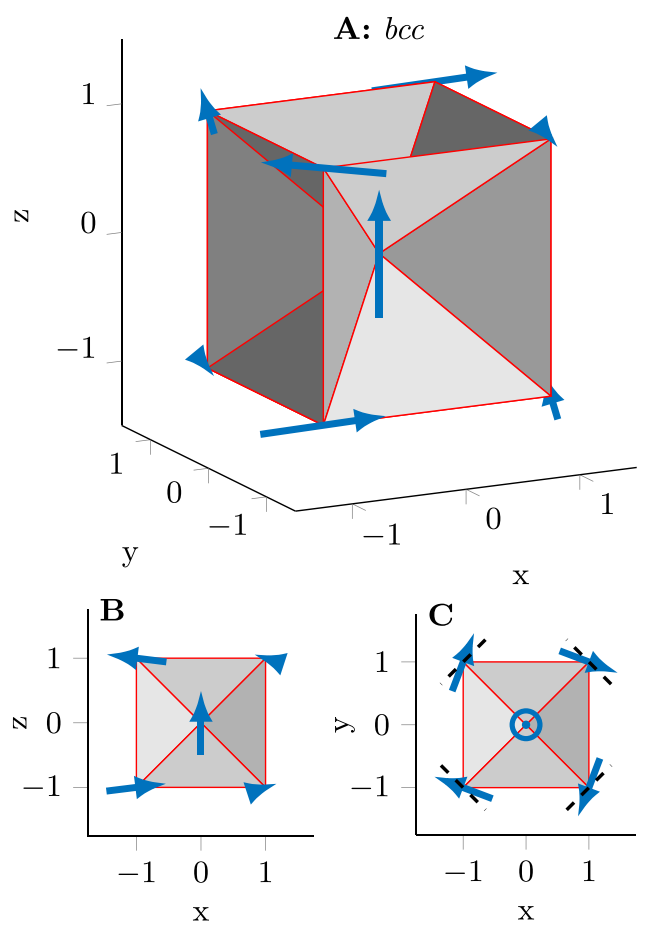

FIG. 5. (a) The ground state of magnetic moments in a $b c c$ structure (blue arrows). The central moment has chosen a direction among the $\langle 100\rangle$ directions. (b) (Side view): The vertex moments all acquire a component in the same direction $(\hat{z})$. (c) (Top view): The vertex moments also acquire an outward component in the $x-y$ plane, such that the moments are no longer tangential to the circumscribed circle (black dashed lines).
$(1+8 \cdot 0.115) \mu=1.92 \mu$. Each vertex moment also acquires an outward component, as seen in Fig. 5(c). All displacements are of such limited magnitude that the counter-rotation of the two layers from the cube ground states is still observed.

\section{Effect of the central moment on the net moment}

For all structures considered, a net moment is generated when a central moment is added as listed in Table I (figures not shown). All structures show a set of particular directions along which the central moment aligns. We find, as for the $b c c$ described above, that the direction of the central moment is perpendicular to the layers that we observed in the panels of Fig. 2, and we observe a change in vertex moment components along the direction of the central moment, i.e., the $\mu_{z}$ direction. The only exception to this trend is found in the square antiprism, which has a metastable state with the central moment towards the center of a square face, but the ground state has the central moment pointing to two points on each triangular face.

The alignment of vertex moments in the direction of the central moment especially gives the smaller structures (tetrahedron, cube, octahedron, and icosahedron) a net moment significantly larger than the strength of the single magnetic moment that is added. The largest net moment is observed in the octahedron, where each vertex moment acquires a component of $0.45 \mu$ in the direction of the central moment, leading to a total net moment of $(1+6 \cdot 0.45) \mu=3.70 \mu$. The dodecahedron [net moment $(1+10 \cdot 0.03+10 \cdot(-0.06))$ $\mu=0.69 \mu \mathrm{l}$ has moments in its inner layers that anti-align with the central moment to yield a negative contribution to the net moment. The icosahedron also acquires this antialignment of the inner layers.

The alignment of vertex moments can be explained by examining the field of a dipole. At the polar angle $\arccos 1 / \sqrt{3}=54.7^{\circ}$, we have $\vec{B}_{\mathrm{dip}, z}=0$, where the $z$-component of the dipolar field changes from parallel to antiparallel to the dipole itself. We adopt the name of the magic angle from the field of nuclear magnetic resonance ${ }^{29}$ and have added a cone at this angle to an icosahedron with a central moment in Fig. 6. The vertex moments of the outer layers are inside the cone and align with the central moment, while those of the inner layers outside the cone align against the central moment.

The angle between the direction of the central moment and a vertex moment in the cube is exactly the magic angle, and the field from the central moment has no component parallel to its direction at the vertex moment positions. However, the field from the central moment does have components that point radially outwards (inwards) at the upper (lower) vertex moment positions. These radial components rotate the previously flux-closed moments, the resulting asymmetry leading to out-of-plane torques between the vertex moments. The resultant effective torque forces the vertex moments out of the $x-y$ plane. Thus, the dipolar field in the $x-y$ direction, rather than in the $z$ direction, is responsible for the observed increase of the net moment.

In general, we attribute the larger net moment for smaller structures (i.e., tetrahedron and octahedron) to the stronger 


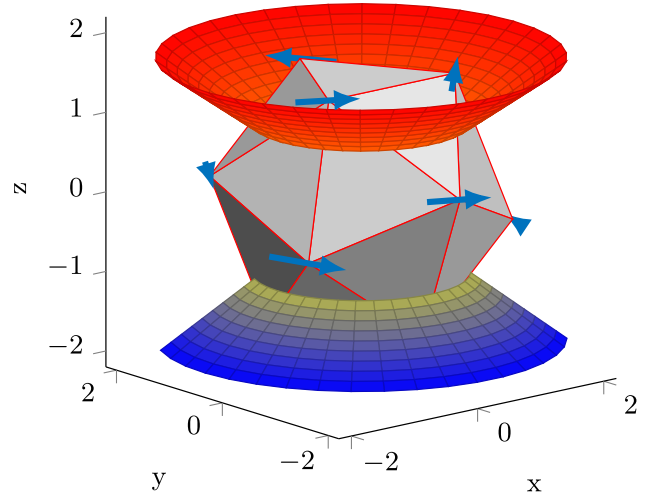

FIG. 6. The ground state of moments (blue arrows) arranged in an icosahedron with a central moment oriented along $\hat{z}$ (not visible), shown with a cone of polar angle $\arccos 1 / \sqrt{3} \approx 54.7^{\circ}$, representative of $B_{\mathrm{dip}, z}=0$. Vertex moments within the cone have a component in the direction of the central moment and vertex moments outside the cone have a component opposite to the central moment.

dipolar interaction (due to the shorter distance) between the central moment and vertex moments.

\section{Effect on the toroidal moment from a central moment}

Under the addition of a central moment, the net moment is no longer zero and the toroidal moment becomes origindependent. In these cases, we have chosen to calculate the toroidal moment from the center of mass of the structures (in Table I).

The tetrahedron and the octahedron lose their toroidal moment when a central moment is added. For the tetrahedron, the central moment results in vertex moment directions reminiscent of those in spin-ice pyrochlore lattices, ${ }^{30}$ with " 2 -in 2-out" configurations. As the vertex moments of one layer all point towards or away from the central moment, their individual components will cancel on a layer-basis, such that the overall toroidal moment is 0 .

The cube structure with a central moment retains its toroidal moment of 0 . For the $b c c$, each layer contributes a toroidal moment in the $\hat{z}$ direction as for the cube, but due to the counter-rotation of the moments, the overall toroidal moment vanishes.

The icosahedron and the dodecahedron have no substantial change in the toroidal moment when a central moment is added, with new values only slightly $(2.4 \%$ and $0.1 \%$, respectively) lower than those for the ground state without a central moment, but otherwise in the same direction. In these structures (i.e., icosahedron and dodecahedron), the distance between vertex and central moments, compared to that of the smaller systems, means that vertex moment directions are only slightly perturbed, and correspondingly, the toroidal moment is only slightly lowered.

\section{Effects on the hysteresis loop from a central moment}

In the simple 2-dimensional case, we have simulated a quasi-static hysteresis loop for the triangle with and without a central moment. The resultant plot for selected field directions can be seen in Fig. 7. Without a central moment, the triangle shows a slight butterfly-opening of the hysteresis loop when the applied field is along a side of the triangle (corresponding to $\hat{x}$ in Fig. 1). The area of this loop is $A=0.07 U_{0} / n$. With the field applied perpendicular to a side of the triangle (i.e., along $\hat{y}$ in Fig. 1), there is no opening of the loop, and hence the hysteresis area $A=0$. With a central moment, the loop of the triangle is open in zero-field. This leads to a hysteresis area of $A_{c}=0.27 U_{0} / n$, when a field is applied perpendicular to a side (corresponding to $\hat{y}$ in Fig. 4). With the field applied along a side of the triangle $(\hat{x}$ in Fig. 4), the hysteresis area is $A_{c}=0.11 U_{0} / n$. We have performed hysteresis loops in field directions at $3^{\circ}$ intervals between the two directions described here, and have found that the triangle with a central moment consistently has a larger area (average $A_{c}=0.16 U_{0} / n$ ) than the triangle without a central moment (average $A=0.04 U_{0} / n$ ), i.e., a 4-fold increase in the average loop area.

We also simulated the hysteresis loops for some Platonic clusters with and without a central moment, with hysteresis loop area per moment $A_{c}$ and $A$, respectively, summarized in Table II. For the tetrahedron and the octahedron, the hysteresis area increases from zero to above $0.5 U_{0} / n$ with the addition of a central moment. The larger structures (cube, icosahedron) have a less significant effect on the hysteresis loop area per moment, with values after the addition of a central moment up to $0.06 U_{0} / n$ and $0.02 U_{0} / n$, respectively. The largest loops are generally found when the magnetic field is in the direction of the central moment in the relaxed cluster. We assume that the partial alignment of the vertex moments to the central moment acts as an effective anisotropy and delays the reversal
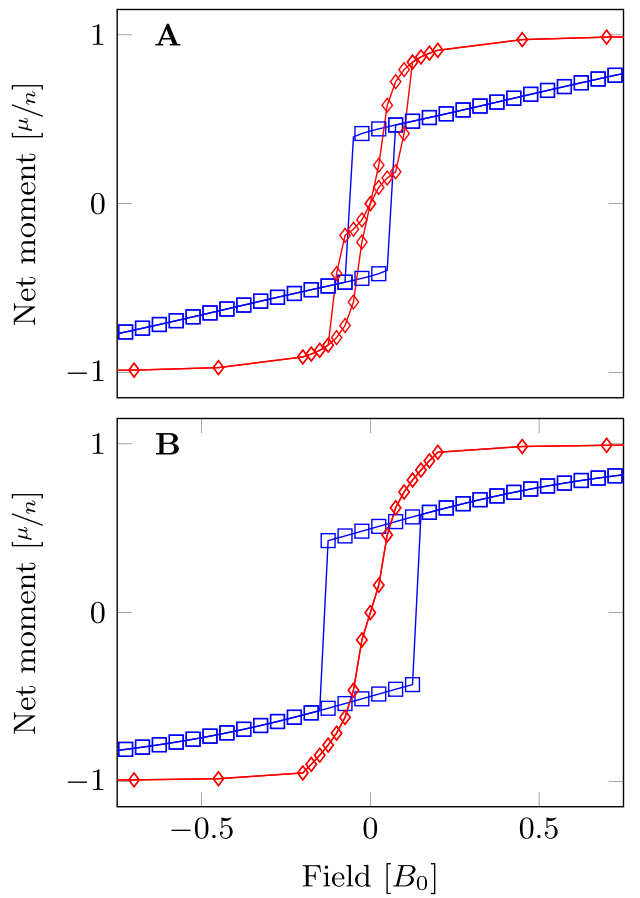

FIG. 7. Hysteresis loop for the triangle without a central moment (cf. Fig. 1, red diamonds) and for the same triangle with a central moment (cf. Fig. 4, blue squares). (a) Field applied in the $\hat{x}$-direction, (b) field applied in the $\hat{y}$ direction. 
TABLE II. The hysteresis loop area per moment in units of $U_{0} / n$ for the listed structures. The areas listed are the smallest and the largest values found along the field directions tested, with the largest areas consistently found along the easy direction of the central moment.

\begin{tabular}{lccc}
\hline \hline Type & $n$ & $A\left(U_{0} / n\right)$ & $A_{\mathrm{c}}\left(U_{0} / n\right)$ \\
\hline Tetrahedron & 4 & $\approx 0-0.01$ & $0.36-0.61$ \\
Octahedron & 6 & $\approx 0$ & $0.15-0.47$ \\
Cube & 8 & $\approx 0-0.01$ & $0.01-0.06$ \\
Icosahedron & 12 & $\approx 0$ & $0.01-0.02$ \\
\hline \hline
\end{tabular}

when the field is reversed. This also explains why we see a stronger effect on the smaller clusters as these have a greater interaction between the central moment and the vertex moments.

\section{CONCLUSIONS}

We have investigated the coupling between the structure and the net moment for clusters of magnetic moments relaxing under dipolar forces exclusively. We find that for threedimensional structures, magnetic dipole moments placed at the vertices of various polyhedra relax to form layers of fluxclosure. These structures have zero remanent moment. We further find that the sense of rotation of the flux-closed layers in these structures is dependent on the packing of moments from layer to layer, such that moments arranged with triangular packing between layers lead to ferrotoroidicity, while those with square packing lead to antiferrotoroidicity.

Our analysis of the same structures, but packed with a single central moment, shows a dramatic increase in the overall magnetic moment, with resulting net moments of up to approximately 4 times the strength of a single moment.

Furthermore, we found that hysteresis loops for a triangle with a central moment has a 4-fold increase in area when averaged over a range of field directions within the plane of the triangle, compared to the hysteresis loop for a triangle without a central moment. The Platonic structures that we have investigated show a similar trend, with the addition of a central moment leading to a significant increase of the hysteresis loop area. This increase is most pronounced for the smaller clusters due to the strong interaction between the central moment and the vertex moments.

These studies show that it is possible to tune the magnetic properties of nanoparticle clusters by structural design. One could assume that, under certain conditions, nanoparticle clusters will form where a central nanoparticle acts as a nucleation center to which other moments adhere, creating clusters mimicking those simulated here. The enhancement of moments and increase in the hysteresis loop area we have found could thus be applicable to real experiments, and dipolar interactions could prove to play a more multi-faceted role than previously thought.

\section{ACKNOWLEDGMENTS}

We acknowledge financial support from Independent Research Fund Denmark.

${ }^{1}$ P. Hugounenq, M. Levy, D. Alloyeau, L. Lartigue, E. Dubois, V. Cabuil, C. Ricolleau, S. Roux, C. Wilhelm, F. Gazeau, and R. Bazzi, J. Phys. Chem. C 116, 15702 (2012).

${ }^{2}$ S. L. Tripp, S. V. Pusztay, A. E. Ribbe, and A. Wei, J. Am. Chem. Soc. 124, 7914 (2002).

${ }^{3}$ M. Klokkenburg, R. P. A. Dullens, W. K. Kegel, B. H. Erné, and A. P. Philipse, Phys. Rev. Lett. 96, 037203 (2006).

${ }^{4}$ J. Ku, D. M. Aruguete, A. P. Alivisatos, and P. L. Geissler, J. Am. Chem. Soc. 133, 838 (2011).

${ }^{5}$ O. Kasyutich, R. D. Desautels, B. W. Southern, and J. van Lierop, Phys. Rev. Lett. 104, 127205 (2010).

${ }^{6}$ R. Skomski and J. M. D. Coey, Phys. Rev. B 48, 15812 (1993).

${ }^{7}$ S. Sun, C. B. Murray, D. Weller, L. Folks, and A. Moser, Science 287, $1989(2000)$

${ }^{8}$ S. Bedanta, T. Eimüller, W. Kleemann, J. Rhensius, F. Stromberg, E. Amaladass, S. Cardoso, and P. P. Freitas, Phys. Rev. Lett. 98, 176601 (2007).

${ }^{9}$ Q. A. Pankhurst, J. Connolly, S. K. Jones, and J. Dobson, J. Phys. D: Appl. Phys. 36, R167 (2003).

${ }^{10}$ C. L. Dennis and R. Ivkov, Int. J. Hyperthermia 29, 715 (2013).

${ }^{11}$ J. M. Luttinger and L. Tisza, Phys. Rev. 70, 954 (1946).

${ }^{12}$ C. Verdes, B. Ruiz-Diaz, S. M. Thompson, R. W. Chantrell, and A. Stancu, Phys. Rev. B 65, 174417 (2002).

${ }^{13}$ R. W. Chantrell, N. Walmsley, J. Gore, and M. Maylin, Phys. Rev. B 63, 024410 (2000).

${ }^{14}$ J. Jordanovic, M. Beleggia, J. Schiøtz, and C. Frandsen, J. Appl. Phys. 118, 043901 (2015).

${ }^{15}$ M. L. Plumer, J. van Lierop, B. W. Southern, and J. P. Whitehead, J. Phys.: Condens. Matter 22, 296007 (2010).

${ }^{16}$ V. Schaller, G. Wahnström, A. Sanz-Velasco, S. Gustafsson, E. Olsson, P. Enoksson, and C. Johansson, Phys. Rev. B 80, 092406 (2009).

${ }^{17}$ N. A. Usov, M. L. Fdez-Gubieda, and J. M. Barandiarán, J. Appl. Phys. 113, 023907 (2013).

${ }^{18}$ F. Ahrentorp, A. Astalan, J. Blomgren, C. Jonasson, E. Wetterskog, P. Svedlindh, A. Lak, F. Ludwig, L. J. van Ijzendoorn, F. Westphal, C. Grüttner, N. Gehrke, S. Gustafsson, E. Olsson, and C. Johansson, in 10th International Conference on the Scientific and Clinical Applications of Magnetic Carriers 10-14 June, 2014, Dresden, Germany [J. Magn. Magn. Mater. 380, 221 (2015)].

${ }^{19}$ A. S. Eggeman, S. A. Majetich, D. Farrell, and Q. A. Pankhurst, IEEE Trans. Magn. 43, 2451 (2007).

${ }^{20}$ M. Allen and D. Tildesley, Computer Simulation of Liquids (Oxford Science Publications/Clarendon Press, 1987).

${ }^{21}$ R. D. Ruth, IEEE Trans. Nucl. Sci. 30, 2669 (1983).

${ }^{22}$ D. J. Evans, Mol. Phys. 34, 317 (1977).

${ }^{23}$ D. R. Stump, G. L. Pollack, and J. Borysowicz, Am. J. Phys. 65, 892 (1997).

${ }^{24}$ C. Ederer and N. A. Spaldin, Phys. Rev. B 76, 214404 (2007).

${ }^{25}$ J. Schonke, T. M. Schneider, and I. Rehberg, Phys. Rev. B 91, 020410 (2015).

${ }^{26}$ B. B. Van Aken, J.-P. Rivera, H. Schmid, and M. Fiebig, Nature 449, 702 (2007).

${ }^{27}$ N. A. Spaldin, M. Fiebig, and M. Mostovoy, J. Phys.: Condens. Matter 20, 434203 (2008).

${ }^{28}$ M. Varón, M. Beleggia, T. Kasama, R. J. Harrison, R. E. DuninBorkowski, V. F. Puntes, and C. Frandsen, Sci. Rep. 3, 1234 (2013).

${ }^{29} \mathrm{~J}$. W. Hennel and J. Klinowski, New Techniques in Solid-State NMR (Springer, 2005), pp. 1-14.

${ }^{30}$ B. C. den Hertog and M. J. P. Gingras, Phys. Rev. Lett. 84, 3430 (2000). 